\title{
Differential Diagnosis between Tumor-Forming Pancreatitis and Pancreatic Cancer by Percutaneous Transhepatic Portography and Selective Direct Pancreatic Venography
}

\author{
HISAFUMI KINOSHITA, HIROYASU IMAYAMA, TOSHIRO OGATA, \\ JUN NAGASHIMA AND SHIGEAKI AOYAGI
}

Department of Surgery, Kurume University School of Medicine, Kurume 830-0011, Japan

\begin{abstract}
Summary: In 32 patients with tumor-forming pancreatitis and 109 patients with pancreatic cancer, the usefulness of percutaneous transhepatic portography (PTP) and selective pancreatic venography (SPV) for differential diagnosis of the two diseases was evaluated. The PTP images were type I in $53.1 \%$, type II in $21.9 \%$, type III in $12.5 \%$, and type IV in $12.5 \%$ of the patients with tumor-forming pancreatitis and type I in $20.2 \%$, type II in $23.9 \%$, type III in $37.6 \%$, and type IV in $18.3 \%$ of the patients with pancreatic cancer. Advanced images (type III or type IV) were observed in more than half the patients with pancreatic cancer. Mild images classified as type II were observed slightly more frequently in the patients with pancreatic cancer, but the differential diagnosis of the two diseases was difficult in patients showing type II PTP images. SPV findings were primarily hypervascularization $(78.1 \%)$ and vasodilation $(68.8 \%)$ in the patients with tumorforming pancreatitis. Although encasement (smooth encasement) was noted in $31.3 \%$, obstruction was found in only $3.1 \%$. In the patients with pancreatic cancer, obstruction was observed in $85.3 \%$, and encasement (irregular encasement) was noted in $78.9 \%$. However, hypervascularization or vasodilatation was infrequent, and the tumor was characteristically imaged as a hypovascular area. PTP and SPV were considered to be useful for the differential diagnosis of tumor-forming pancreatitis and pancreatic cancer.
\end{abstract}

Key words tumor-forming pancreatitis, pancreatic cancer, percutaneous transhepatic portography, selective direct pancreatic venography

\section{INTRODUCTION}

Although the differential diagnosis of tumor-forming pancreatitis and pancreatic cancer has been facilitated by improvements in various imaging techniques such as ultrasonography (US), endoscopic ultrasonography (EUS), intraductal ultrasonography (IDUS), endoscopic retrograde cholangiopancreatography (ERCP), computed tomography (CT), magnetic resonance imaging (MRI), and angiography and the development of tumor markers including CA19-9, Dupan-2, and Span-1, it still poses consid- erable difficulty in some cases. In this study, the usefulness of percutaneous transhepatic portography (PTP) and selective pancreatic venography (SPV) for the differential diagnosis of tumor-forming pancreatitis and pancreatic cancer was evaluated.

\section{SUBJECTS AND METHODS}

\section{Patients}

The subjects were 32 patients with tumor-forming pancreatitis and 109 patients with pancreatic 
cancer who underwent PTP and SPV between February, 1980 and December, 1998 (Table 1). The portal images obtained by PTP were classified into type I (normal), type II (displaced), type III (stenosed), type IV (obstructed). The images of the pancreatic vein obtained by SPV were also examined with regard to displacement, stenosis, obstruction, hypervascularization, vasodilation, and collateral formation.

\section{Technique}

As shown in Fig. 1a, PTP was normally per-

TABLE 1

Tumor-forming pancreatitis and pancreatic cancer were examined with PTP and SPV

\begin{tabular}{lc}
\hline \multicolumn{1}{c}{ disease } & No. of patients \\
\hline Tumor-forming pancreatitis & 32 \\
Pancreatic cancer & 109 \\
\hline Total & 141 \\
\hline
\end{tabular}

(February, 1980 to December, 1998) formed by the double catheter method (sheath method): First, an intrahepatic portal branch (usually the bifurcation of the anterosuperior and anteroinferior branches of the right anterior segmental ramus) was punctured with a 21-23 G (usually $21 \mathrm{G}$ ) PTC needle under ultrasound guidance, and a 0.0018-inch J-shaped guide wire was inserted. Next, a 5.0-5.5 French sheath was advanced nearly to the portal trunk, a 4.5-5.0 French catheter was inserted, and the portal vein (PV), superior mesenteric vein (SMV), splenic vein (SV), and the target pancreatic veins (anterior superior pancreaticoduodenal vein (ASPDV), posterior superior pancreaticoduodenal vein (PSPDV), and dorsal pancreatic vein (DPV)) were visualized. Also, as shown in Fig. 1b, the balloon catheter method (obstructive venography) was performed in combination: A 4.0-5.0 French balloon catheter was inserted by the double catheter method, the venous trunk was obstructed using the balloon, and the pancreatic vein was visualized via the collaterals.

\section{a. Double catheter technique}

1

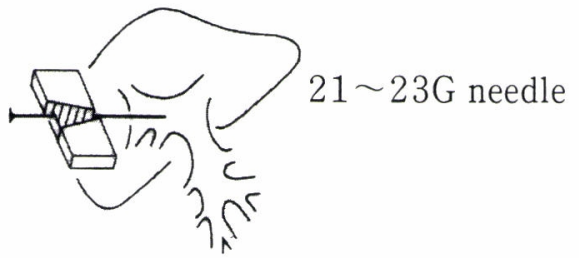

3

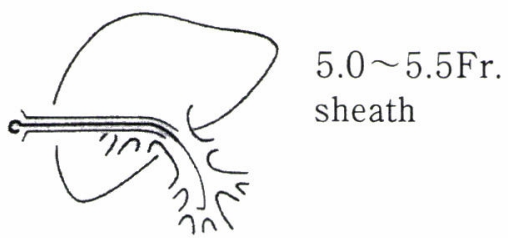

2

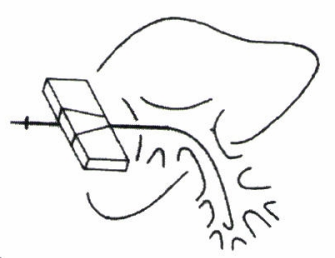

0.018 inch J type guide wire

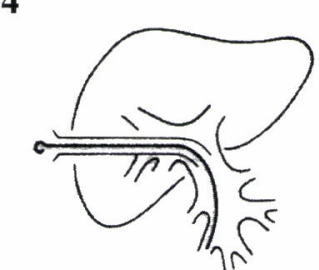

$4.0 \sim 5.0 \mathrm{Fr}$. catheter

\section{b. Balloon catheter technique}

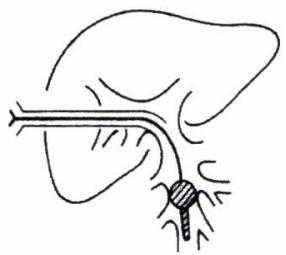

$4.0 \sim 5.0 \mathrm{Fr}$.

balloon catheter

Fig. 1. Technique for PTP and SPV 


\section{RESULTS}

Portal vein images of tumor-forming pancreatitis and pancreatic cancer

In the 32 patients with tumor-forming pancreatitis, the PTP images were type I in 17, type II in 7, type III in 4, and type IV in 4. Type I accounted for $53.1 \%$, and advanced findings of type III and type IV were observed in $25.0 \%$. In the 109 patients with pancreatic cancer, the PTP images were type I in 22, type II in 26 , type III in 41 , and type IV in 20 . Type I accounted for $20.2 \%$, and type III and type IV together accounted for $56.0 \%$. Type II was observed in $21.9 \%$ of the patients with tumor-forming pancreatitis and $23.9 \%$ of those with pancreatic cancer (Table 2).

Pancreatic vein images in tumor-forming pancreatitis and pancreatic cancer

In the 32 patients with tumor-forming pancreatitis, hypervascularization was noted in $25(78.1 \%)$, vasodilation in $22(68.8 \%)$, encasement (smooth encasement) in $10(31.3 \%)$, displacement in 3 $(9.4 \%)$, and obstruction and collateral formation in 1 each $(3.1 \%)$. Hypervascularization and vasodilation were primary findings. Although stenosis was observed occasionally, obstruction was rare. In the 109 patients with pancreatic cancer, obstruction was observed in $93(85.3 \%)$, encasement (irregular encasement) in $86(78.9 \%)$, collateral formation in $23(21.1 \%)$, displacement in $21(19.3 \%)$, vasodilation in $11(10.1 \%)$, and hypervascularization in $6(5.5 \%)$. Obstruction and stenosis were primary findings, hypervascularization and vasodilation were infrequent, and the tumor was characteristically visualized as a hypovascular area (Table 3 ).

Case 1: Tumor-forming pancreatitis (Fig. 2) Since celiac arteriography showed encasement and interruption in branches and the trunk of the PSPDA and a hypovascular area in the head of pancreas, cancer of the head of pancreas was suspected. PTP showed no abnormality in the SMV to the portal trunk. GCT

TABLE 2.

Findings of portal venogram in tumor-forming pancreatitis and pancreatic cancer

\begin{tabular}{|c|c|c|c|c|}
\hline & $\begin{array}{c}\text { Type I } \\
\text { normal }\end{array}$ & $\begin{array}{c}\text { Type II } \\
\text { focal stenosis }\end{array}$ & $\begin{array}{c}\text { Type III } \\
\text { circular stenosis }\end{array}$ & $\begin{array}{c}\text { Type IV } \\
\text { total obstruction }\end{array}$ \\
\hline $\begin{array}{l}\text { Tumor-forming } \\
\text { pancreatitis } \\
\qquad(\mathrm{n}=32)\end{array}$ & $\begin{array}{c}17 \\
(53.1)\end{array}$ & $\begin{array}{c}7 \\
(21.9)\end{array}$ & $\begin{array}{c}4 \\
(12.5)\end{array}$ & $\begin{array}{c}4 \\
(12.5)\end{array}$ \\
\hline $\begin{array}{l}\text { Pancreatic } \\
\text { cancer } \\
\qquad(n=109)\end{array}$ & $\begin{array}{c}22 \\
(20.2)\end{array}$ & $\begin{array}{c}26 \\
(23.9)\end{array}$ & $\begin{array}{c}41 \\
(37.6)\end{array}$ & $\begin{array}{c}20 \\
(18.3)\end{array}$ \\
\hline
\end{tabular}

TABLE 3.

Findings of pancreatic venogram in tumor-forming pancreatitis and pancreatic cancer

\begin{tabular}{lcc}
\hline & $\begin{array}{c}\text { Tumor-forming pancreatitis } \\
(\mathrm{n}=32)\end{array}$ & $\begin{array}{c}\text { Pancreatic cancer } \\
(\mathrm{n}=109)\end{array}$ \\
\hline displacement & $3(9.4)$ & $21(19.3)$ \\
encasement & $10(31.3)$ & $86(78.9)$ \\
obstruction & $1(3.1)$ & $93(85.3)$ \\
vasodilatation & $25(78.1)$ & $6(5.5)$ \\
hypervascularization & $22(68.8)$ & $11(10.1)$ \\
collateral formation & $1(3.1)$ & $23(21.1)$ \\
\hline * including duplication finding & \multicolumn{2}{c}{()$: \%$}
\end{tabular}




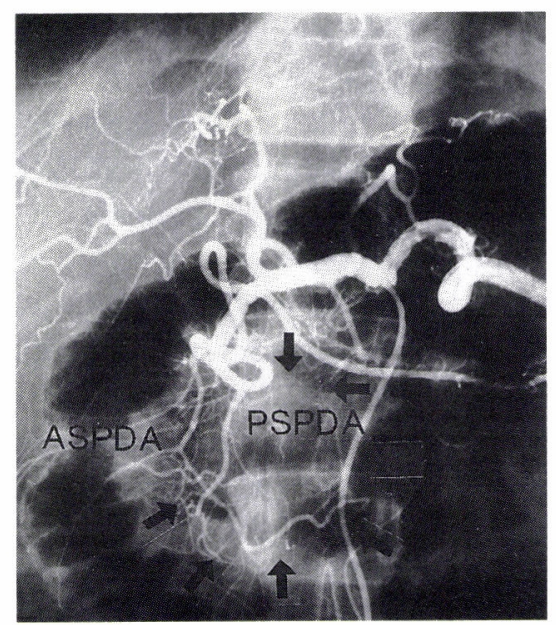

a

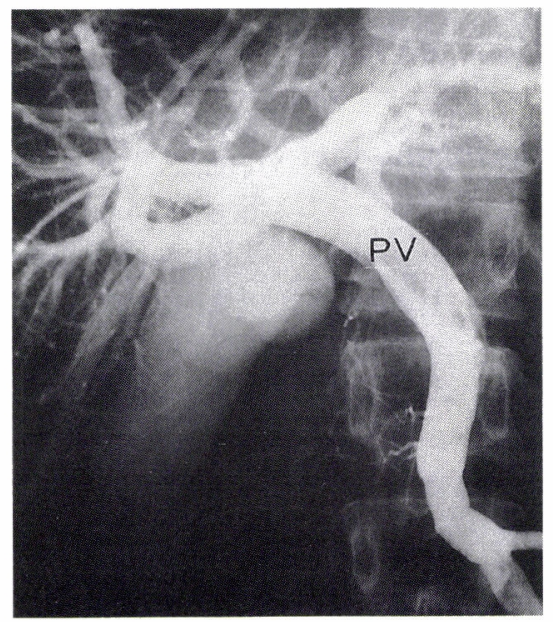

b

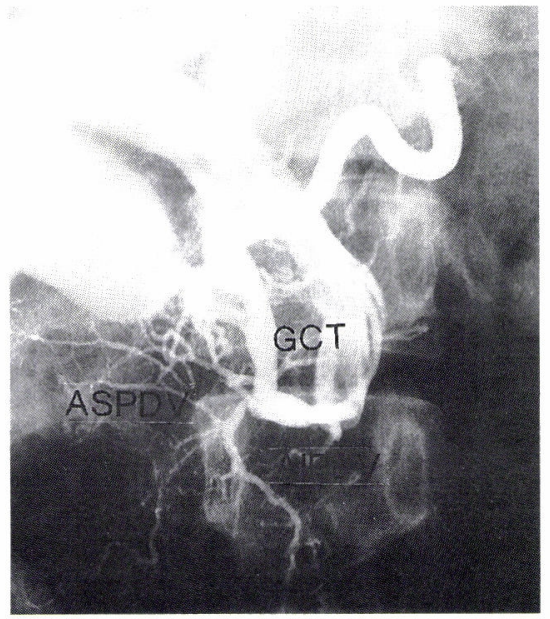

C

Fig. 2. Tumor-forming pancreatitis.

a: Celiac arteriography showed encasement and interruption in the trunk and branches of the PSPDA and a hypovascular area in the head of the pancreas.

b: PTP showed no particular abnormality from the SMV to the PV trunk (PTP: Type I).

c: GCT clearly visualized the pancreatic vein and showed no encasement or interruption.

PSPDA : posterior superior pancreaticoduodenal artery

ASPDA : anterior superior pancreaticoduodenal artery

ASPDV : anterior superior pancreaticoduodenal vein

AIPDV : anterior inferior pancreaticoduodenal vein

GCT : gastrocolic trunk SMV : superior mesenteric vein PV : portal vein

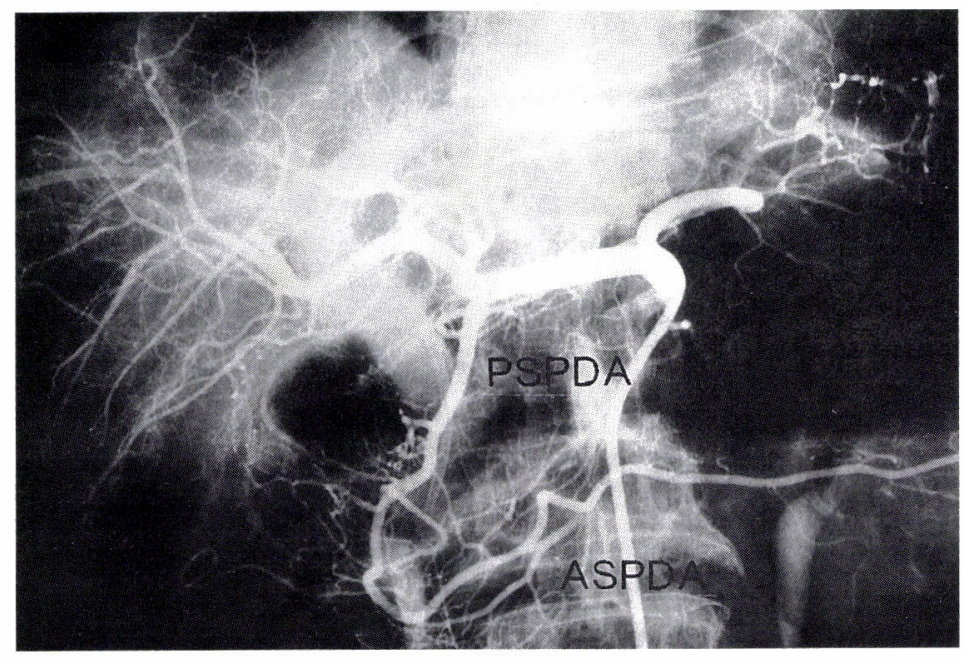

a

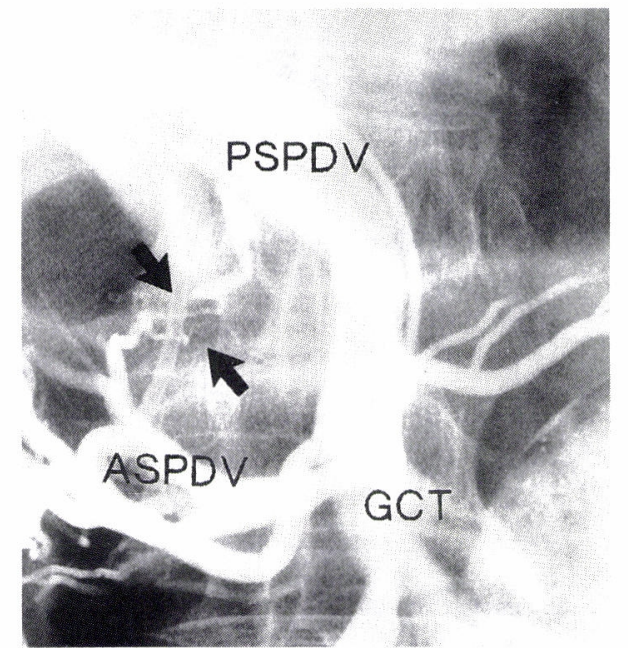

b

Fig. 3. Pancreatic head cancer.

a: Celiac arteriography clearly delineated the arteries of the pancreatic arcades and their branches, and no abnormality was noted.

b: PTP and SPV showed no particular abnormality from the SMV to the PV trunk (PTP: Type I), but obstruction and collateral formation were noted at the communication between the ASPDV and PSPDV (arrow). 
clearly delineated the pancreatic vein without encasement or interruption so that a diagnosis of tumorforming pancreatitis was made, and the patient was observed without surgery.

Case 2: Pancreatic cancer (Fig. 3) Celiac arteriography clearly visualized the pancreaticoduodenal arterial arcades and their branches, and no abnormality was noted, suggesting tumor-forming pancreatitis. PTP and SPV showed no particular abnormality in the SMV to the portal trunk, but obstruction and collateral formation were noted at the communication between the ASPDV and PSPDV. Therefore, a diagnosis of cancer of the head of pancreas was made, and pancreaticoduodenectomy was performed.

\section{DISCUSSION}

Although tumor-forming pancreatitis has been regarded as a clinical manifestation of chronic pancreatitis, it is presently recognized as a unique variation of chronic pancreatitis that presents images that resemble those of pancreatic cancer. The differential diagnosis of tumor-forming pancreatitis and pancreatic cancer is difficult even by the extensive use of various imaging techniques, and surgery is performed in many cases without a definitive diagnosis. Preoperative differentiation of the two disorders is an important problem for surgeons in determination of whether the pancreas should be resected or not. Among findings on various imaging modalities, US, EUS, and IDUS findings that contribute to the differential diagnosis of tumor-forming pancreatitis and pancreatic cancer include the morphology, margin, and boundary of the mass, internal echo, echo level, duct structure in the mass, penetrating duct sign, and images of the pancreatic duct caudal to the mass [1-4]. The usefulness of US angiography by intra-arterial infusion of $\mathrm{CO}_{2}$ gas has also been reported [5]. ERCP findings that contribute to the differentiation between the two diseases are the nature of obstruction site in the pancreatic duct, lack of branches of the pancreatic duct, morphology and degree of dilation of the caudal pancreatic duct, and the length and continuity of the stenotic area [6]. Ikeda et al. [7] reported the necessity of balloon catheter ERP-compression study, and Yasuda et al. [8] suggested that cytological examination of pancreatic juice and histological examination by biopsy under ERCP guidance and the puncture cytology under EUS guidance are effective means for this purpose. On CT, the presence or absence of calcification, whether the main pancreatic duct or its branches are identified or not in the mass, whether the mass shows homogeneous intense staining in an early phase of dynamic CT or not, and whether the size of the mass changes in a relatively short period or not have been suggested to be points of differentiation between the two diseases [9]. Neff et al. [10] observed that the image intensity of the mass is the most reliable finding for the differential diagnosis and reported that tumor-forming pancreatitis is stained equally with the surrounding tissues while pancreatic cancer shows homogeneous and faint staining. Concerning discriminating findings on abdominal angiography, bending, meandering, beads-like deformation, and smooth encasement of arteries in and around the pancreas are reported to be characteristic of tumor-forming pancreatitis, but obstruction, irregular encasement, abrupt angulation, and displacement of arteries and a hypovascular area around abnormal arteries have been described as characteristics of pancreatic cancer [11]. Suyama et al. [12] observed that visualization of arteries in the pancreas is indispensable for the angiographic diagnosis of pancreatic tumors and that ultra-selective angiography of the gastroduodenal artery, anterior and posterior arcades, dorsal pancreatic artery, and major pancreatic artery is necessary. However, there are false positive and false negative findings in any examination, and the differential diagnosis is often difficult. To overcome this problem, selective pancreatic venography has been performed at our department since 1980 as a means of differential diagnosis of the two disorders. Since PTP was introduced by Bierman et al. [13], it has been applied clinically and yielded a number of reports. The literature is rich concerning the angiographic diagnosis of pancreatic disorders, but most of it is about evaluation of findings on arterial phase and transarterial portography, and there have been few reports on pancreatic venograms obtained by PTP. PTP and SPV produce clearer venous images than transarterial portography and can delineate even small pancreatic veins. Portograms obtained by PTP were type I in more than half the patients with tumorforming pancreatitis but showed advanced changes classified as type III or type IV in a majority of patients with pancreatic cancer. However, mild changes classified as type II were observed in $21.9 \%$ of those with tumor-forming pancreatitis and $23.9 \%$ of those with pancreatic cancer, and differentiation of the two diseases in patients showing type II portal images was considered to be difficult. Portograms are considered to present only mild findings in many 
patients with tumor-forming pancreatitis, because the findings are due to inflammatory changes, but marked findings in many patients with pancreatic cancer, because they are due to direct infiltration of cancer itself. Miyoshi et al. [14] observed that arterial portography (AP) is advantageous in that it can be performed as an extension of arteriography but that PTP allows not only reliable interpretation of images that are unreadable by AP but also differentiation of benign and malignant tumors and judgment of the extent of the tumor despite its complex procedure.

On pancreatic venography, hypervascularization and vasodilation were primary findings in tumorforming pancreatitis, and although smooth encasement was observed, obstruction was rare. In Case 1, arteriographic findings suggested cancer of the head of pancreas, but pancreatic venography clearly delineated the pancreatic vein, and a diagnosis of tumorforming pancreatitis was made. No changes are observed today, 6 years after the diagnosis, and the patient has returned to his job. Findings on pancreatic venography in pancreatic cancer were primarily obstruction and irregular encasement, and collateral formation was notable, but hypervascularization or vasodilation was rare. The tumor was characteristically visualized as a hypovascular area. In case 2 , no abnormality was noted in arteries on arteriography, and tumor-forming pancreatitis was suspected. However, a diagnosis of cancer of the head of pancreas was made, because pancreatic venography showed obstruction of the pancreatic vein and collateral formation, and surgery was performed. Concerning the pancreatic vein, Mano [15] suggested vasodilatation, hypervascularization, and encasement of rami as characteristic angiographic findings of tumor-forming pancreatitis and described that the local blood flow was relatively intact in this disease compared with ischemic pancreatic cancer. The frequency of obstruction of large veins was low in tumor-forming pancreatitis but high in pancreatic cancer, probably because of predominantly fibrotic changes in the former but infiltrative growth in the latter. These observations suggest that PTP and SPV are useful as means of differential diagnosis of tumor-forming pancreatitis and pancreatic cancer.

\section{REFERENCES}

1. Hayashi Y, Nakazawa S, Kimoto E, Naito Y, and Morita K. Clinicopathologic analysis of endoscopic ultrasono- grams in pancreatic mass lesion. Endoscopy 1989; 21:121-125.

2. Akimoto S, Shimura N, Itoh T, and Hanyu F. Differential diagnosis between pancreatitis and pancreatic cancer by ultrasonography. The Biliary and Pancreas 1984; 5:957966. (in Japanese)

3. Furukawa T, Naitoh Y, Tsukamoto Y, Hirooka Y, Katoh $\mathrm{T}$ et al. Differential diagnosis between pancreatic cancer and mass forming pancreatitis by intraductal ultrasoundsystem. Pancreas 1994; 9:452-460.

4. Mukai H, Yasuda K, and Nakashima M. Endoscopic ultrasonography in the differential diagnosis between pancreatic cancer and pseudo-tomorous pancreatitis. Diagnostic Imaging of the Abdomen 1993; 13:13-20. (in Japanese)

5. Nakao T. Differential diagnosis between tumor-forming pancreatic cancer and tumor-forming pancreatitis by contrast enhanced ultrasonography. J Jpn Panc Soc 1992; 7:629-636. (in Japanese)

6. Rohrmann CA, Silvis SE, and Vennes JA. The significance of pancreatic ductal obstruction in differential diagnosis of the abnormal endoscopic retrograde pancreatogram. Radiology 1976; 121:311-314.

7. Ikeda S, Maeshiro K, Matsumoto S, Okamoto K, Miyazaki $\mathrm{R}$ et al. ERCP diagnosis of mass-forming pancreatitis. Diagnostic Imaging of the Abdomen 1995; 15:583-589. (in Japanese)

8. Yasuda K, Nakashima M, Cho E, Hayakumo $T$, Mochizuki $\mathrm{N}$ et al. Differential diagnosis of tumorforming pancreatitis and pancreatic carcinoma by endoscopic procedures. The Biliary Tract and Pancreas 1999; 20:297-304. (in Japanese)

9. Leutmer PH, Stephens DH, and Ward EM. Chronic pancreatitis; Reassessment with current CT. Radiology $1989 ; 171: 353-357$.

10. Neff CC, Simeone JF, Wittenberg J, Mueller PR, and Ferrucci Jr JT. Inflammatory pancreatic masses; problems in differentiating forcal pancreatitis from carcinoma. Radiology 1984; 150:35-38.

11. Nebeser RA, and Pollard JJ. A critical evaluation of selective celiac and superior mesenteric angiography in the diagnosis of particularly malignant tumor: Facts and "artifacts." Radiology 1967; 89:1017-1027.

12. Suyama M, Ariyama J, Satoh K, Nagaiwa J, Kubokawa $\mathrm{Y}$ et al. Diagnosis of tumor forming pancreatitis by angiography. Diagnostic Imaging of the Abdomen 1995; 15:614-619. (in Japanese)

13. Bierman HR, Kelly KH, White LP, Coblent ZA, and Fissher A. Trancehepatic venous catheterization and venography. JAMA 1955; 158:1331-1334.

14. Miyoshi A, Mano M, Ueki T, Nakayama T, Koga M et al. The study of portalvenography in the disease of pancreatoduodenal region. The Biliary Tract and Pancreas 1982; 3:525-533. (in Japanese)

15. Mano M. Angiographic study of the portal and pancreatic veins in disease of the pancreatic head. J Kurume Med Assoc 1986; 49:878-889. (in Japanese) 